\title{
Tumor Diameter for Prediction of Recurrence, Disease Free and Overall Survival in Endometrial Cancer Cases
}

\author{
Taylan Senol, Mesut Polat, Enis Ozkaya, Ates Karateke
}

\begin{abstract}
Aims: To analyse the predictors of recurrence, disease free survival and overall survival in cases with endometrial cancer. Materials and Methods: A total of 152 women diagnosed with endometrial cancer were screened using a prospectively collected database including age, smoking history, menopausal status, body mass index, CA125, systemic disorders, tumor histology, tumor grade, lymphovascular space invasion, tumor diameter, cervical involvement, myometrial invasion, adnexal metastases, positive cytology, serosal involvement, other pelvic metastases, type of surgery, fertility sparing approach to assess their ability to predict recurrence, disease free survival and overall survival. Results: In ROC analyses tumor diameter was a significant predictor of recurrence (AUC:0.771, P<0.001). The optimal cut off value was 3.75 with $82 \%$ sensitivity and $63 \%$ specificity. In correlation analyses tumor grade $(r=0.267, p=0.001)$, tumor diameter $(r=0.297, p<0.001)$ and the serosal involvement $(r=0.464$, $\mathbf{p}<0.001)$ were found to significantly correlate with the recurrence. In Cox regression analyses when some different combinations of variables included in the model which are found to be significantly associated with the presence of recurrence, tumor diameter was found to be a significant confounder for disease free survival $(O R=1.2(95$ CI,1.016-1.394, $P=0.031)$. On Cox regression for overall survival only serosal involvement was found to be a significant predictor $(\mathrm{OR}=20.8(95 \% \mathrm{CI}$ 2.4-179.2, $\mathrm{P}=\mathbf{0 . 0 0 6})$. In univariate analysis of tumor diameter $>3.75$ $\mathrm{cm}$ and the recurrence, there was $14(21.9 \%)$ cases with recurrence in group with high tumor diameter where as only $3(3.4 \%)$ cases group with smaller tumor size (Odds ratio:7.9 (95 \% CI 2.2-28.9, p<0.001). Conclusions: Although most of the significantly correlated variables are part of the FIGO staging, tumor diameter was also found to be predictor for recurrence with higher values than generally accepted.
\end{abstract}

Keywords: Endometrial cancer - tumor diameter - overall survival - disease free survival

Asian Pac J Cancer Prev, 16 (17), 7463-7466

\section{Introduction}

Endometrial carcinomas are the most common malignancy of female genital tract in developed countries (Siegel et al., 2012). More than $70 \%$ of women present with disease confined to the uterus, with the remainder having more advanced disease (Creasman et al., 2006). The main treatment of endometrial cancer is surgical, which includes total hysterectomy, bilateral salpingooophorectomy, peritoneal washings, and pelvic and paraaortic lymphadenectomy (Panici et al., 2008). The indication and extent of lymph node dissection in the surgical management of endometrial cancer remain highly controversial (Panici et al., 2008, Kitchener et al., 2009, Seracchioli et al., 2010). There is a consensus about the necessity of complete surgical staging for high risk endometrial cancers due to higher risk of nodal metastasis, the need for pelvic and para-aortic lymph node dissection with complete surgical staging for the low risk endometrial cancer is still debated passionately (Kumar et al., 2011). There has been several factors defined that is associated with recurrence, survival or both (Dunn et al., 2014).

The aim of this study was to analyse the predictors of recurrence, disease free survival and overall survival in cases with endometrial cancer.

\section{Materials and Methods}

We performed a study among 152 women with endometrial cancer treated at the Department of Gynecological oncology of the Zeynep Kamil Maternity and Children's Training and Research Hospital, from January 2010 to December 2014. Inclusion criteria were endometrial cancer confirmed by pre-operative endometrial sampling, who underwent surgical therapy, and appropriate data obtained from database, under regular clinical follow up; exclusion criteria were positive endoservical sampling indicating cervical adenocancer, subjects under hormon replacement therapy, loss of follow up, incomplete adjuvant therapy, incomplete staging surgery. The staging surgery procedure (pelvic-para-aortic lymphadenectomy) was performed based on the FS report. 


\section{Taylan Senol et al}

At our institute, the staging surgery procedure is performed with the following criteria: Grade 1 or 2 endometrioid carcinoma with $\mathrm{MI}>50 \%$; grade 3 tumors; tumors with cervical extension; clear cell or serous carcinoma; tumor size $>2 \mathrm{~cm}$. Depending on the presence of high-risk factors for lymph nodal metastases at FS related to the Mayo Clinic algorithm and on the surgeon's clinical evaluation, pelvic and, eventually, paraaortic LND was performed. Permanent microscopic evaluation of histology, tumor grade, and stage were made according to the World Health Organization and FIGO classifications, respectively. The surgical procedure consisted of an exploratory laparotomy or laparoscopy, peritoneal washings for cytology, hysterectomy, and bilateral salpingo-oophorectomy. After the uterus was removed, it was sent for FS evaluation to determine the depth of myometrial invasion and tumor grade.

The staging surgery procedure (pelvic-para-aortic lymphadenectomy) was performed based on the FS report. age, smoking, menopasusal status, body mass index, CA125, systemic disorders, tumor histology, tumor grade, lenfovascular space invasion, tm diameter, cervical involvement, myometrial invasion, adnexal metastases, positive cytology, serosal involvement, other pelvic metastases, type of surgery, fertility sparing approach were all analysed to predict recurrence, disease free survival and overall survival in cases with endometrial cancer

\section{Statistical analaysis}

All data was analysed by SPSS version 15 . All data was summarized by descriptive statistics, correlation analyses was used to show associations, multivaraite regression was used to show adjusted associations, roc analysis was used to calculate predictive value, sensitivity and the specificity. And cox regression was used to determine predictors for survival. $\mathrm{P}<0.05$ was accepted to be statistically significant.

\section{Results}

Some demographic and the clinical features of the study population were summarized in Table 1 . In roc analyses, tm diameter was a significant predictor for the recurrence (AUC:0.771, $\mathrm{P}<0.001$, Figure 1 ). Optimal cut off value was 3.75 with $82 \%$ sensitivity, $63 \%$ specificity. In correlation analyses tumor grade $(r=0.267$, $\mathrm{p}=0.001)$, tumor diameter $(\mathrm{r}=0.297, \mathrm{p}<0.001)$ and the serosal involvement $(\mathrm{r}=0.464, \mathrm{p}<0.001)$ were found to significantly correlated with the recurrence. On the other hand serozal involvement was found to be significantly associated with the mortality $(\mathrm{r}=0.306, \mathrm{p}<0.001)$. In linear regression analyses tumor diameter (beta coefficient $=0.186, \mathrm{p}=0.014$ and the serosal involvement

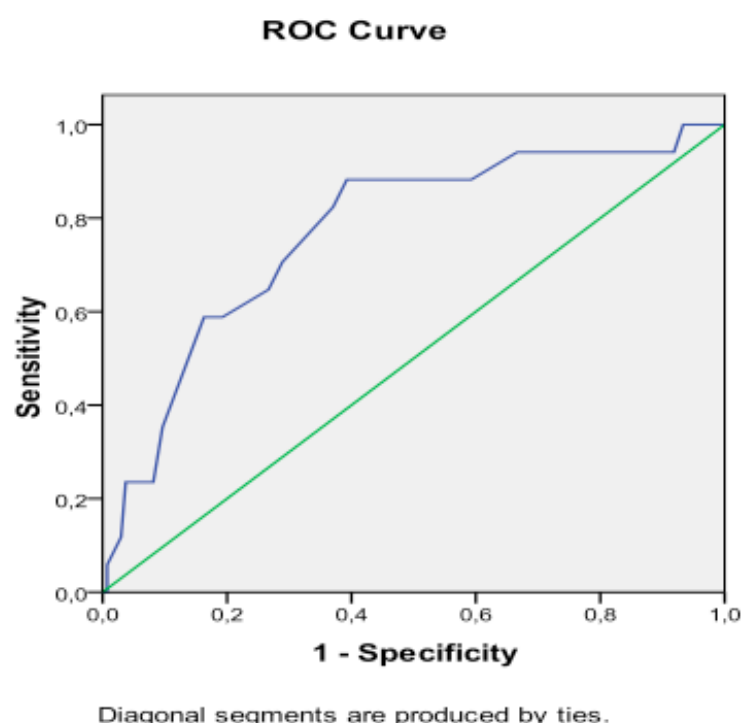

Figure 1. ROC Curve of Tumor Diameter to Predict Recurrence

Table 1. Some Demographic and the Clinical Features of all Study Population.

\begin{tabular}{|c|c|c|c|c|c|}
\hline & $\mathrm{N}$ & Minimum & Maximum & Mean & Std. Deviation \\
\hline Age & 152 & 29 & 80 & 56.3 & 9.9 \\
\hline Gravidity & 152 & 0 & 17 & 3.5 & 2.5 \\
\hline Parity & 152 & 0 & 9 & 2.6 & 1.7 \\
\hline BMI & 152 & 17.9 & 48.4 & 31.3 & 5.3 \\
\hline CA125 & 152 & 2.41 & 4339 & 82.4 & 464.1 \\
\hline Tumor diameter, $\mathrm{cm}$ & 152 & 0.1 & 12 & 3.6 & 2.6 \\
\hline Total lymph nodes & 152 & 0 & 73 & 19.5 & 16.3 \\
\hline No of metastatic pelvic lymph node & 152 & 0 & 15 & 0.3 & 1.6 \\
\hline No of metastatic pa lymph nodes & 152 & 0 & 3 & 0.1 & 0.5 \\
\hline total metastatic lymph nodes & 152 & 0 & 17 & 0.4 & 1.9 \\
\hline Duration till recurrence & 152 & 3 & 30 & 15 & 8.4 \\
\hline Total time of follow up period & 152 & 0 & 57 & 27.5 & 16.8 \\
\hline
\end{tabular}

BMI:Body mass index

Table 2. Cox Regression Analyses Result to Predict Disease Free Survivial

\begin{tabular}{|c|c|c|c|c|c|c|c|c|}
\hline & \multirow[t]{2}{*}{ B } & \multirow[t]{2}{*}{$\mathrm{SE}$} & \multirow[t]{2}{*}{ Wald } & \multirow[t]{2}{*}{ Df } & \multirow[t]{2}{*}{ Sig. } & \multirow[t]{2}{*}{$\operatorname{Exp}(B)$} & \multicolumn{2}{|c|}{$95.0 \% \mathrm{CI}$ for $\operatorname{Exp}(\mathrm{B})$} \\
\hline & & & & & & & Lower & Upper \\
\hline $\mathrm{TD}$ & 0.174 & 0.081 & 4.673 & 1 & 0.031 & 1.19 & 1.016 & 1.394 \\
\hline Histology & 0.157 & 0.08 & 3.824 & 1 & 0.051 & 1.17 & 1 & 1.368 \\
\hline Serosa & 1.832 & 0.602 & 9.269 & 1 & 0.002 & 6.248 & 1.921 & 20.323 \\
\hline
\end{tabular}

TD:Tumor diameter 
(beta coefficient $=0.381, \mathrm{p}<0.001$ ) were found to be significantly associated with the recurrence. In cox regression analyses when some different combinations of variables included in the model which are found to be significantly associated with the presence of recurrence tm diameter is found to be significant confounder for disease free survival $(\mathrm{OR}=1.2$ (95 CI,1.016-1.394, $\mathrm{P}=0.031$, Table $2)$. In cox regression for overall survival only the serosal involvement was found to be a significant predictor $(\mathrm{OR}=20.8$ (95 \% CI 2.4-179.2, $\mathrm{P}=0.006)$, tumor diameter was not found to be predictor for overall survival $(\mathrm{p}>$ $0.05)$. In univariate analysis of tumor diameter $>3.75$ $\mathrm{cm}$ and the recurrence, there were $14(21.9 \%)$ cases with recurrence in group with high tumor diameter where as only $3(3.4 \%)$ cases group with smaller tumor size (Odds ratio:7.9 (95\%CI 2.2-28.9, $\mathrm{p}<0.001)$.

\section{Discussion}

In this study, we aimed to analyse the 152 women with endometrial cancer treated at the Department of Gynecological Oncology of the Zeynep Kamil Women and Children's HealthTraining and Research Hospital, from January 2010 to December 2014 to figure out the predictors for recurrence, ovarall and disease free survival. Our analyses of the data revealed that CA125, histology and the serosal involvement are significantly associated with the recurrence and when cox regression model is established to predict disease free survival, histology, serosal involvement and the tumor diameter were found to be predictors for the DFS. Only serosal involvement found to be associated with the ovearall survival. An article published in 2005 indicated that, tumor size correlated with extrauterine disease, but it was found to be an independent prognostic variable for nodal metastases however authors concluded that, tumor size does not appear to be an independent predictor of recurrence (Shah et al., 2014).

In another study published in 2013 conluded that, In high-grade endometrial cancers, tumor involvement of the lower uterine segment and tumor size $>2 \mathrm{~cm}$ were associated with pelvic nodal disease, and lower uterine segment involvement was also significantly associated with para-aortic nodal disease. With regard to recurrence no association was found between lower uterine segment involvement or tumor size $>2 \mathrm{~cm}$ and recurrence (Doll et al., 2014). In our study although no association was found between the tumor size with the pelvic lymph or paraaortic lymph node metastases it was found to be associated with the disease free survival and the cut off value was found to be $3.75 \mathrm{~cm}$ with acceptable $82 \%$ sensitivity and $63 \%$ specificity. Two recently published studies assessed the predictive value of tumor diameter for the prognosis of endometrial cancer, first study indicate that anteroposterior tumor diameter greater than $2 \mathrm{~cm}$ predicts deep myometrial invasion, and craniocaudal planes tumor diameter greater than $4 \mathrm{~cm}$ predicts lymph node metastases. Tumor size is a strong prognostic factor in endometrial carcinomas. Preoperative tumor mea-surements based on MRI may potentially improve preoperative risk stratification models and thus enable better tailored surgical treatment in endometrial cancer (Ytre-Hauge et al., 2015). This data shows the prognostic value of three dimentions of tumor in endometrial cancer cases. The second recently published study consistent with the our results showed that, tumor size was an independent predictor of lymph node metastasis and disease-specific survival in patients with endometrioid endometrial cancer grossly confined to the uterus. Tumor $>5 \mathrm{~cm}$ was a predictor of disease-specific survival but no difference in outcome was noted between tumor $>2-5 \mathrm{~cm}$ and tumor $\leq 2 \mathrm{~cm}$ (Mahdi et al., 2014). Study on frozen section in endometrial cancer surgery revealed that accuracy of frozen section for myometrial invasion, grade and low risk prediction significantly increased with decreasing tumor diameter and study indicate the cut off value of $3 \mathrm{~cm}$ tumor size that frozen section can be accurate enough to exclude lymph node metastases, our cut off was $3.75 \mathrm{~cm}$ and possible explanation of this size leading to poor prognosis is inaccurate frozen section and incomplete surgery accordingly (Yanazume et al., 2011). Tumor diameter has shown to be predictor for lymph node dissemination in study by Alhilli et al. (AlHilli et al., 2013). Also there are publications about the low sensitivity of intraoperative gross examination for detecting deep myometrial invasion and cervical invasion in early-stage endometrial cancer to be used alone. Study emphasized the three dimensional evaluation as alternative methods including intraoperative frozen section analysis, preoperative three dimensional ultrasound, and preoperative magnetic resonance imaging (Sethasathien et al., 2014). Survival analyses of a small study poplutaion consisted of 84 patients from Iran revealed that he prognosis of endometrial cancer was fair but strongly varied by stage and grade, and moderately varied by histology and age (Binesh et al., 2014). According to accumulated data and results of our study, we suggest not to measure two diamentional evaluation of tumor but also three dimensional measurement of the tumor size, this drawback in determination of tumor size may cause inconsistency for predictive value of tumor size for endometrial cancer prognosis.Further studies are needed to assess predictive value of tumor volume for the prognosis, this promising assessment may help selecting patients for more aggresive or conservative management. And our study showed that inconsistency may be due to the acceptance of cut off value to be $2 \mathrm{~cm}$, as it was shown in our study higher values are more predictive. In conclusion, although most of the significantly correlated variables are part of the FIGO staging, tm diameter was also found to be predictor for recurrence with higher values than generally accepted and our study suggest to assess three diamentional assessment in furher studies for prediction of prognosis.

\section{References}

AlHilli MM, Podratz KC, Dowdy SC, et al (2013). Preoperative biopsy and intraoperative tumor diameter predict lymph node dissemination in endometrial cancer. Gynecol Oncol, 128, 294-9.

Benedetti Panici P, Basile S, Maneschi F, et al (2008). Systematic pelvic lymphadenectomy vs. no lymphadenectomy in early- 
stage endometrial carcinoma: randomized clinical trial. $J$ Natl Cancer Inst, 100, 1707-16.

Binesh F, Akhavan A, Behniafard N, et al (2014). Endometrial adenocarcinoma: clinicopathologic and survival characteristics in Yazd, Iran. Asian Pac J Cancer Prev, 15, 2797-801.

Creasman WT, Odicino F, Maisonneuve P, et al (2006). Carcinoma of the corpus uteri. FIGO 26th Annual Report on the results of treatment in ynecological cancer. Int $J$ Gynaecol Obstet, 95, 105-43.

Dunn EF, Geye H, Platta CS, et al (2014). Predictive factors of recurrence following adjuvant vaginal cuff brachytherapy alone for stage I endometrial cancer. Gynecol Oncol, 133, 494-8.

Doll KM, Tseng J, Denslow SA, et al (2014). High-grade endometrial cancer: revisiting the impact of tumor size and location on outcomes. Gynecol Oncol, 132, 44-9.

Kitchener H, Swart AM, Qian Q, et al (2009). Efficacy of systematic pelvic lymphadenectomy in endometrial cancer (MRC ASTEC trial): a randomised study. Lancet, 373, 125-36.

Kumar S, Bandyopadhyay S, Semaan A, et al (2011). The role of frozen section in surgical staging of low risk endometrial cancer. PLoS One, 6, 21912.

Mahdi H, Munkarah AR, Ali-Fehmi R, et al (2014). Tumor size is an independent predictor of lymph node metastasis and survival in early stage endometrioid endometrial cancer. Arch Gynecol Obstet. Dec 31. [Epub ahead of print]

Seracchioli R, Solfrini S, Mabrouk M, et al (2010). Controversies in surgical staging of endometrial cancer. Obstet Gynecol Int $\mathbf{1 8 1 9 6 3 .}$

Sethasathien P, Charoenkwan K, Siriaunkgul S, et al (2014). Accuracy of intraoperative gross examination of myometrial invasion in stage I-II endometrial cancer. Asian Pac J Cancer Prev, 15, 7061-4.

Shah C, Johnson EB, Everett E, et al (2005). Does size matter? Tumor size and morphology as predictors of nodal status and recurrence in endometrial cancer. Gynecol Oncol, 99, 564-70.

Siegel R, Naishadham D, Jemal A, et al (2012). Cancer statistics. CA Cancer J Clin, 62, 10-29.

Yanazume S, Saito T, Eto T, et al (2011). Reassessment of the utility of frozen sections in endometrial cancer surgery using tumor diameter as an additional factor. Am J Obstet Gynecol, 204, 531 .

Ytre-Hauge S, Husby JA, Magnussen IJ, et al (2015). Preoperative tumor size at MRI predicts deep myometrial invasion, lymph node metastases, and patient outcome in endometrial carcinomas. Int J Gynecol Cancer, 25, 459-66. 